\title{
Experimental and Numerical Examination of the Fastening System's Rail Clip Type SB-4
}

\author{
Ludomir J. Jankowski ${ }^{1, a}{ }^{*}$, Jaroslaw Filipiak ${ }^{1, b}$, Malgorzata Zak ${ }^{1, c}$ \\ Krzysztof Kruszyk ${ }^{2, d}$ \\ ${ }^{1}$ Wroclaw University of Science and Technology, Faculty of Mechanical Engineering, \\ 50-370 Wroclaw, Poland \\ ${ }^{2}$ Vossloh SKAMO Sp. z 0.0., 63-460 Nowe Skalmierzyce, Poland

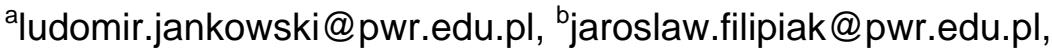 \\ cmalgorzata.a.zak@pwr.edu.pl, dkrzysztof.kruszyk@vossloh-skamo.pl
}

Keywords: Rail Fastening System, Spring Clip, Photoelastic Coating Technique, Strain Gauges Technique

\begin{abstract}
The paper presents the results of an experimental and numerical analysis of the effort of the elastic clip type SB-4 VK, during the process of its manual fastening on an anchor embedded in the pre-stressed concrete sleeper. The techniques of photoelastic coating and electrical resistance strain gauges were applied and a numerical simulation of this process was carried out to determine the level of the material effort of the attachment key component. The obtained results of the experiments and numerical calculations showed an acceptable level of the clip material effort.
\end{abstract}

\section{Introduction}

The use of elastic systems for fixing rails to railway track made of pre-stressed concrete sleepers is a solution that increases comfort and safety of driving. Systems such as, for example, the type W [1], e-Clip [2], FASTCLIP [3], as well as the SB system [4], are characterized by high vibration damping efficiency, while ensuring the necessary requirements resulting from applicable international standards [5], including the rail pressure to the ground. The basic element of such systems are elastic clips of different geometry. Due to the specific geometry, the embedding of clips in anchors fixed in sleepers generates complex stress states, hence the assessment of the state of effort during this, usually one-off, working phase is important for an assessment of the fatigue strength of the clamp.

The spring clip of the SB-4 VK fastening system is made of spring steel PN-EN $50 \mathrm{~S} 2$ (1.5024) and is used to clamp the UIC60 (60E1) rail. The clip is attached to the pre-tensioned concrete sleepers using a suitably shaped anchor - Fig. 1a. The manual fastening procedure involves the use of the lever shown in Fig. 1b. It is also possible to remove the clip to change the force applied to the lever.

Experimental tests were carried out on the randomly selected clips provided by the manufacturer, after verifying (in accordance with company standards) the quality of the produced series of springs. The clips were embedded in the anchor by hand following the procedure provided by the manufacturer [6]. 

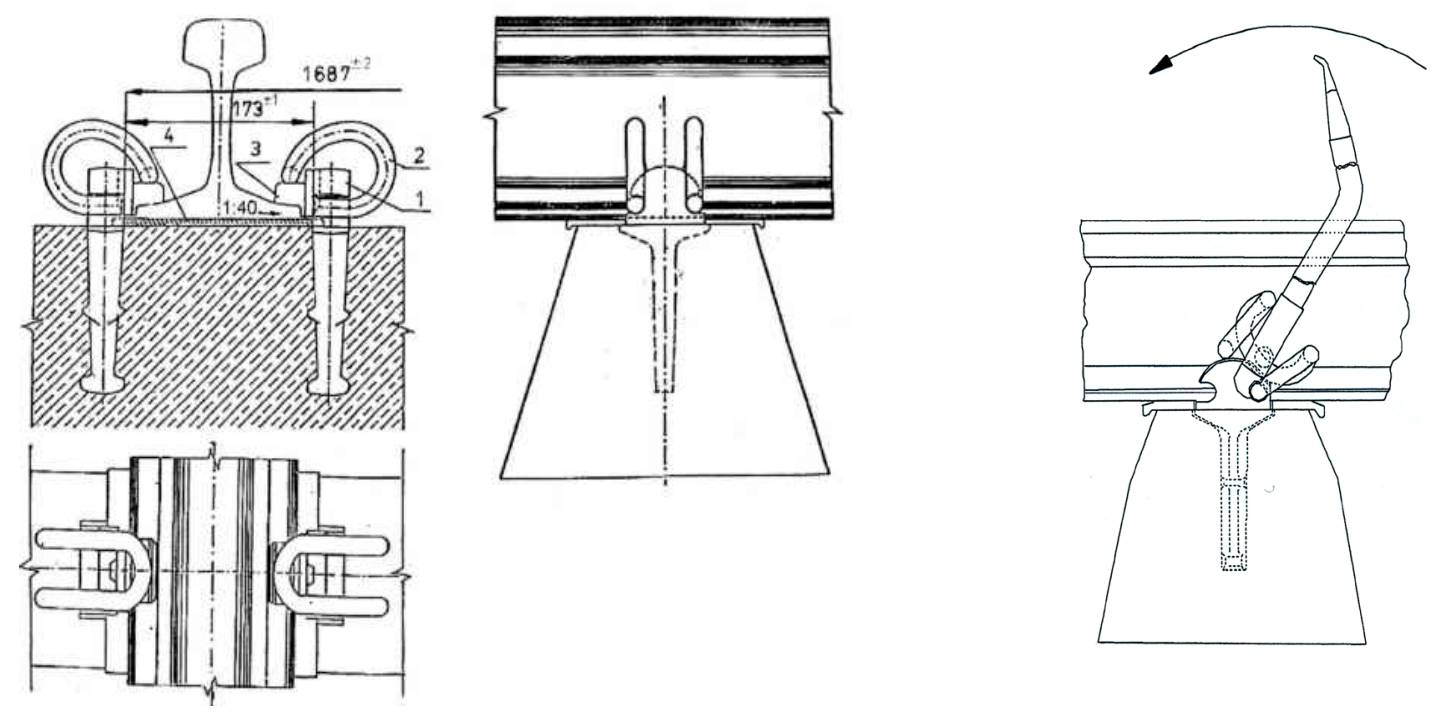

Fig. 1. SB fastening system of the UIC 60 rail [6]: a) 1 - anchor, 2 - spring clip, 3 - electroinsulating pressure pad, 4 - elastic rail pad, b) scheme of the SB-4 VK clip manual deposition

\section{Experimental investigations}

Basing on the preliminary analysis of the clip's work during its fastening, a decision was made to use the photoelastic coating technique $[7,8]$ and the resistance strain gauge method $[7,8]$ to measure the strains during the process characterized by the largest deformation of the geometry of this attachment element. The tested clips were fastened, according to the assembly instructions, on a system provided by the manufacturer simulating fixing the rail to the underlay. Practically, with one exception of the concrete railway sleeper, all elements of the SB4 VK fastening system and their geometry meet the conditions specified in EN 13481-2 and TSI (Technical Specification for Interoperability) [5].

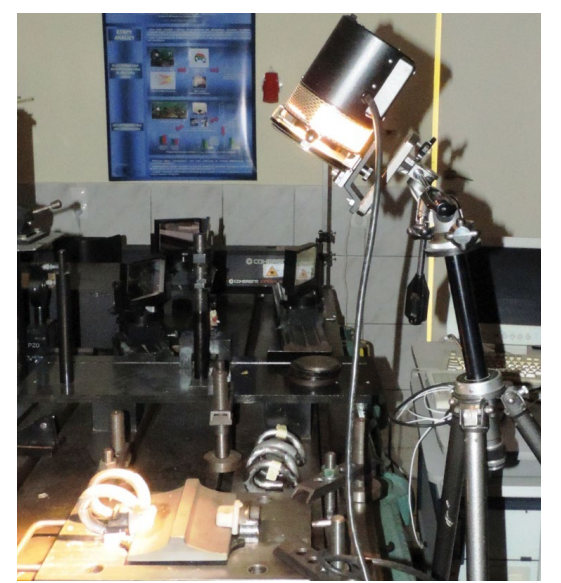

Fig 2. View of the measurement system

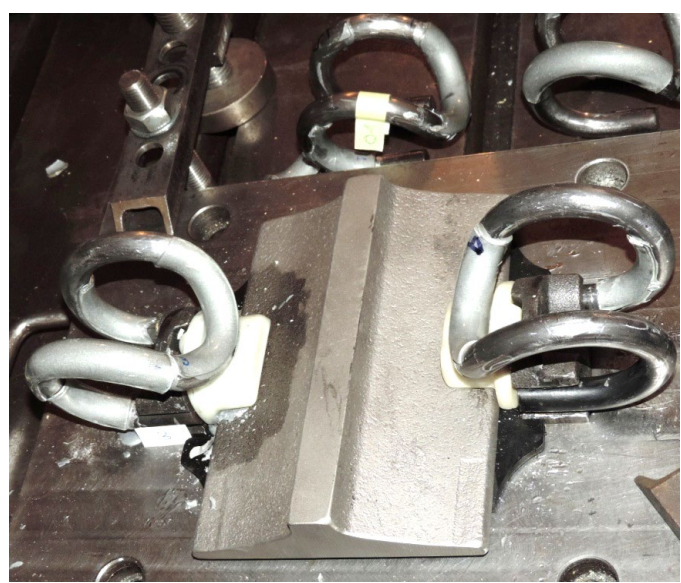

Fig. 3. SB4 clips on a stand

The view of the measurement system during the measurement with the photoelastic coating technique is shown in Fig. 2, and the view of the rail fastening system with the clip covered with photoelastic coating - in Fig. 3. Due to the application of the optical strain measurement method, only the UIC 60 rail bottom part was affixed, which facilitated the observation of optical effects 
in the layer glued to the previously cleaned surface of the clip. In the case of the discussed measurements, a coating of $t_{c}=2.0 \mathrm{~mm}$ thickness was used. Due to the shape of the examined surfaces of the clip, the coating was made in the form of so-called contour plates.

A composition based on an epoxy resin was used as the coating material. The plates formed on the surface of the clip were glued to the surfaces to be tested, using a reflective glue prepared on the basis of the above-mentioned composition with an addition of aluminum dust. For the purpose of quantitative analysis of the state of deformation, the value of isochromatic fringe pattern was determined, as $f_{\varepsilon}=1.58 \times 10^{-3}[-]$.

The measurements were carried out using a V-type reflection polariscope - Model 031, by Photoelastic Vishay Inc., recording photoelastic images with a digital camera. Due to the dynamics of the clip fastening process, the video mode was the basic recording mode. Sample frames of the registered changes of the image of full-order isochromatic fringes during the process of fastening as a function of standardized time $T$ from the interval $[0,1]$ are presented below in Fig. 4.
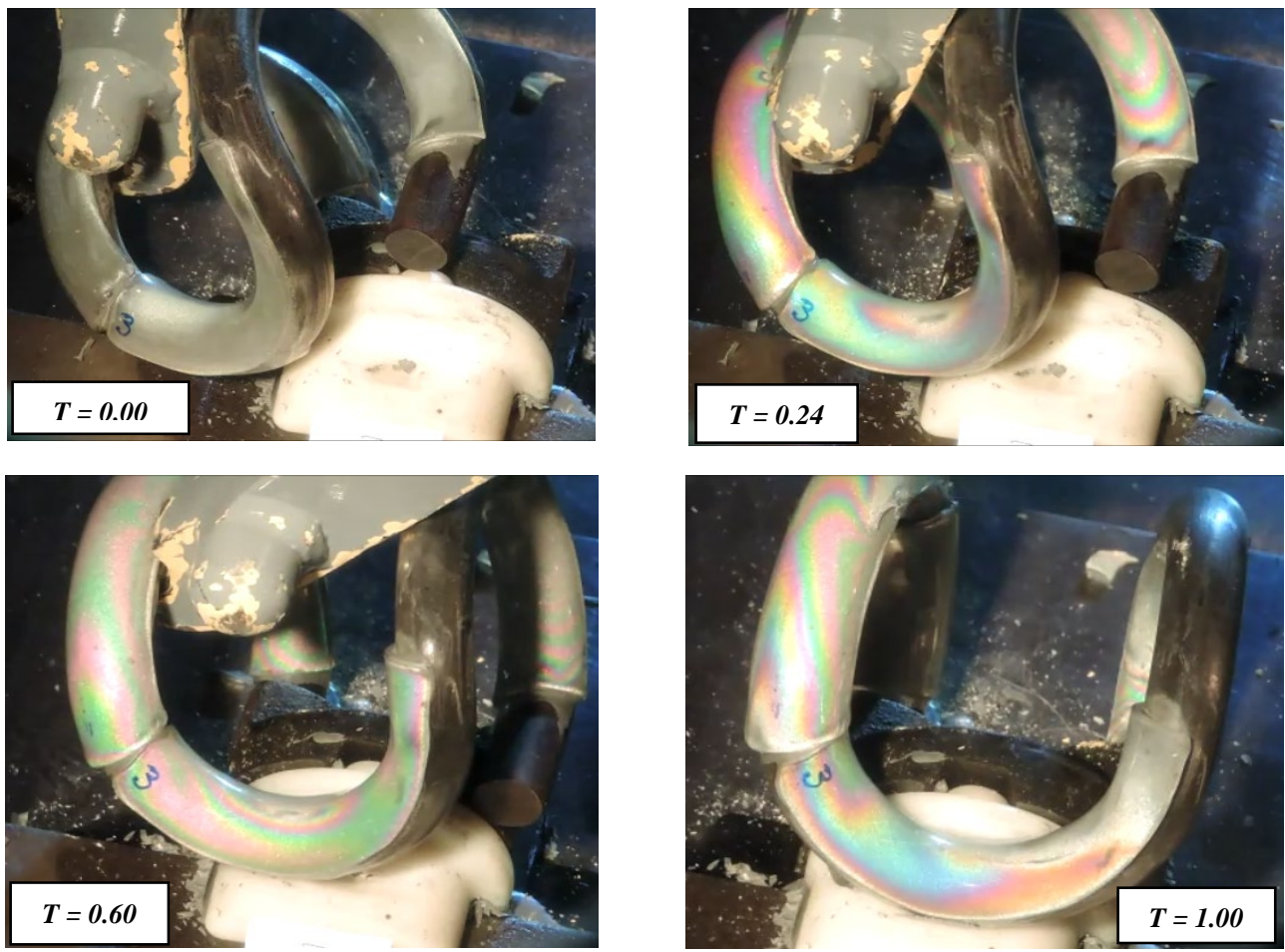

Fig. 4. Views of the full-order isochromatic fringes during fastening SB4 clip

In this case of photoelastic coating technique research, the isochromatic fringe corresponds to the difference of the principal strains:

$$
\varepsilon_{1}-\varepsilon_{2}=N f_{\varepsilon}
$$

where: $N$ - isochromatic fringe order, $f_{\varepsilon}$ - strain-value of the isochromatic fringe.

Due to the dominance of the deformations caused by the bending of individual fragments of the clip, a correction factor $[7,8]$ was introduced, which captures the influence of the strain gradient on the coating thickness:

$$
\left(\varepsilon_{1}-\varepsilon_{2}\right)^{o}=\left(\varepsilon_{1}-\varepsilon_{2}\right)^{c} \cdot K_{b}=N f_{\varepsilon} K_{b}
$$


In the case under consideration, the value of $K_{b}$ is: $K_{b}=0.897$.

The difference of the principal stresses, for $E^{o}=2.05 \times 10^{5} \mathrm{MPa}$ and $v^{o}=0.3$, is determined by the equation:

$$
\left(\sigma_{1}-\sigma_{2}\right)^{o}=\frac{E^{o}}{\left(1+v^{o}\right)} N f_{\varepsilon} K_{b}=141.45 \cdot N[M P a]
$$

(3)

While fastening the clip, the maximum values of the isochromatic fringe order range of $N \cong 6.5$ were observed, which corresponds to the difference of the principal stresses of $920 \mathrm{MPa}$.

The preliminary, qualitative analysis of the registered images of isochromatic fringes enabled the identification of the locations of the resistance strain-gauges, which is shown in Fig. 5.

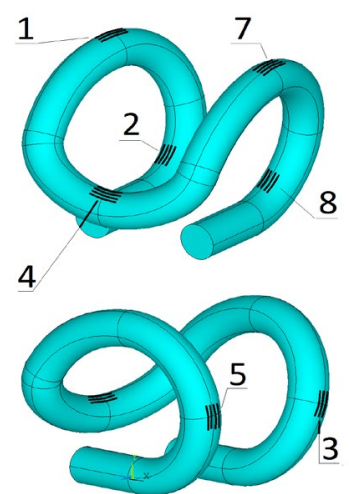

Fig. 5. Location of the

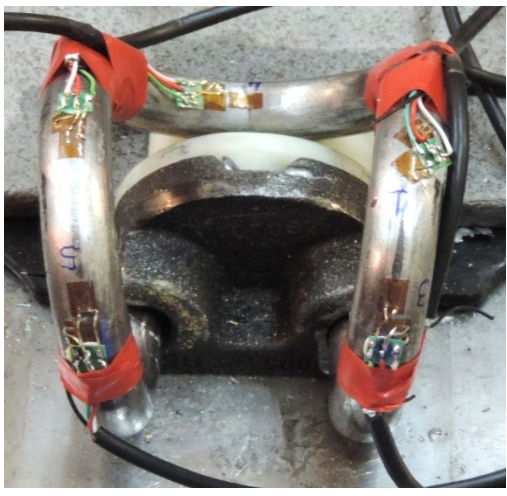

Fig. 6. View of the clip with strain-gauges resistance strain-gauges

Foil strain gauges with a measuring base $\mathrm{a}=3 \mathrm{~mm}$ were used. They were glued in selected places in the Wheatstone half-bridge system [8]. The measurements were carried out with the Stretton 0316E measurement system allowing simultaneous sampling of 16 measurement channels. The view of the clip with glued gauges is shown in Fig. 6. An example of the changes registered in the stress value during the clip fastening is presented in Fig. 7.

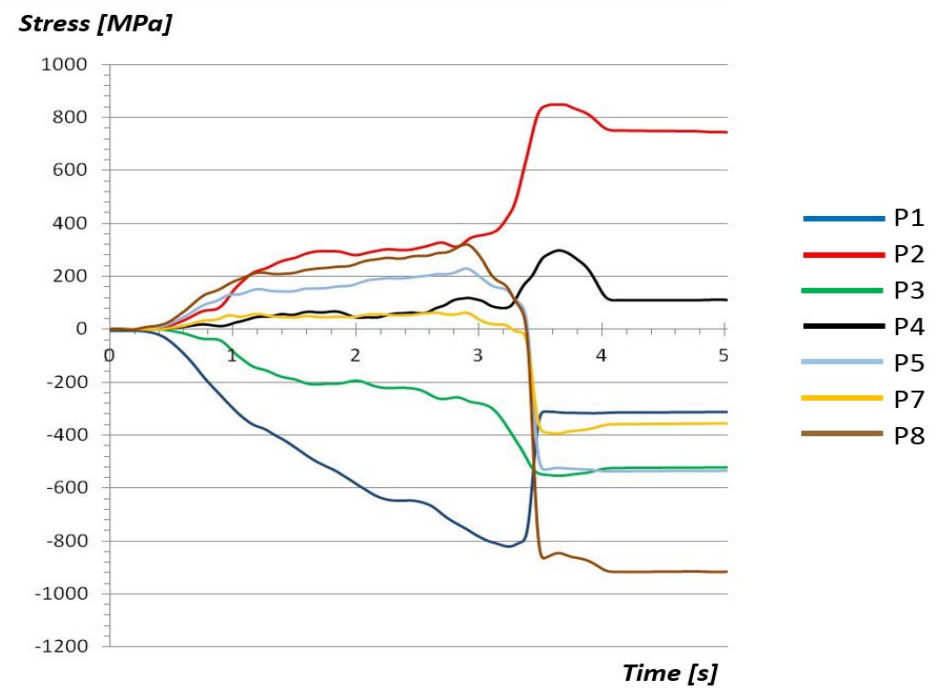

Fig. 7. Changes of the normal stress in the selected (P1-P8) points 


\section{Numerical simulations}

Numerical simulations of the stress state in the spring clip SB4 were carried out using the finite element method (FEM) in the ANSYS Workbench 16.2 environment. The geometric model of the SB4 clip and anchor (excluding its stem) is shown in Fig. 8.

The tests included both static and dynamic simulations, allowing to determine the distribution of stresses for the following cases:

a) bending (stretching) the free arms of the SB4 clip with a force of $6.8 \mathrm{kN}$,

b) operation with a bending force of $14 \mathrm{kN}$ on the central arc of the SB4 clip,

c) fastening the SB4 clip on the anchor.
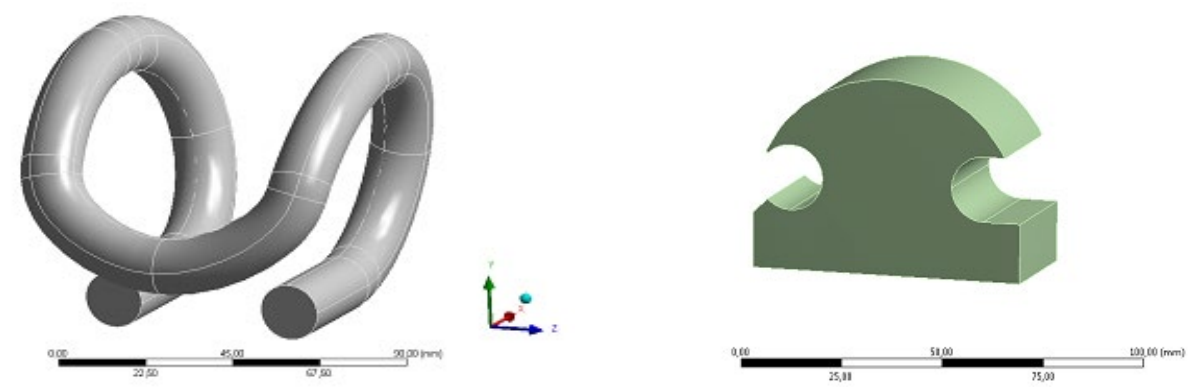

Fig. 8. Geometric models of the clip and anchor head

The following material parameters of spring steel 50S2 (1.5024) were adopted for numerical calculations: density $\rho=7850 \mathrm{kgm}^{-3}$, Young's modulus $E=205 \mathrm{GPa}$, Poisson's ratio $v=0.3$, elastic limit $R_{e}=1250 \mathrm{~N} / \mathrm{mm}^{2}$.

The static simulations carried out for standard clip loads (cases a) and b)) showed permissible values of maximum stresses, i.e. $\sigma_{\max }<R_{e}[9]$.

In case of c) a simulation of clip fastening on the anchor was carried out, using the software for the analysis of fast-changing phenomena employing the LS-DYNA finite element method. The geometric model of the initial position of the clip SB4 with respect to the anchor is shown in Fig. 9.
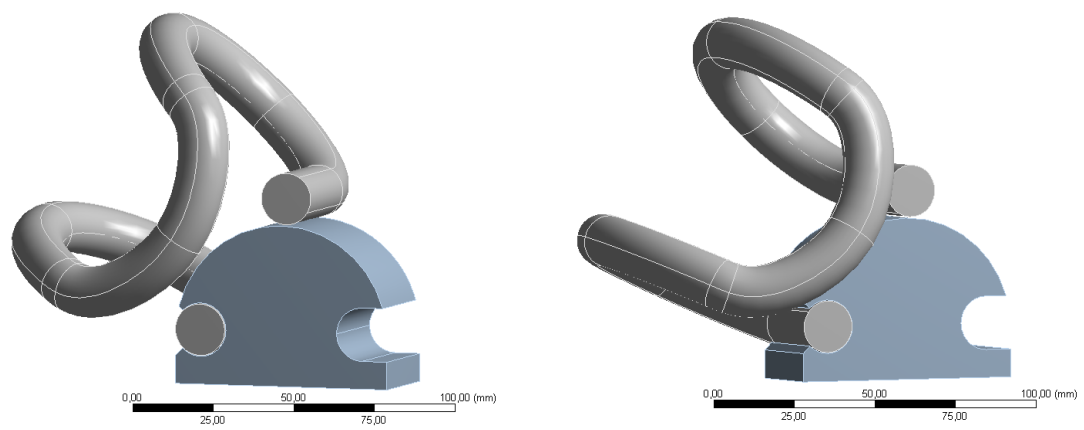

Fig. 9. Geometric model of the initial position of the clip (for dynamic simulation of the clip fastening)

An example of the distribution of the stress difference contours $\left(\sigma_{y}-\sigma_{z}\right)$, obtained in the final stage of fastening the clip, is shown in Fig. 10a, while Figure 10b shows the contours of the reduced stress (von Mises) for the same clip position. 

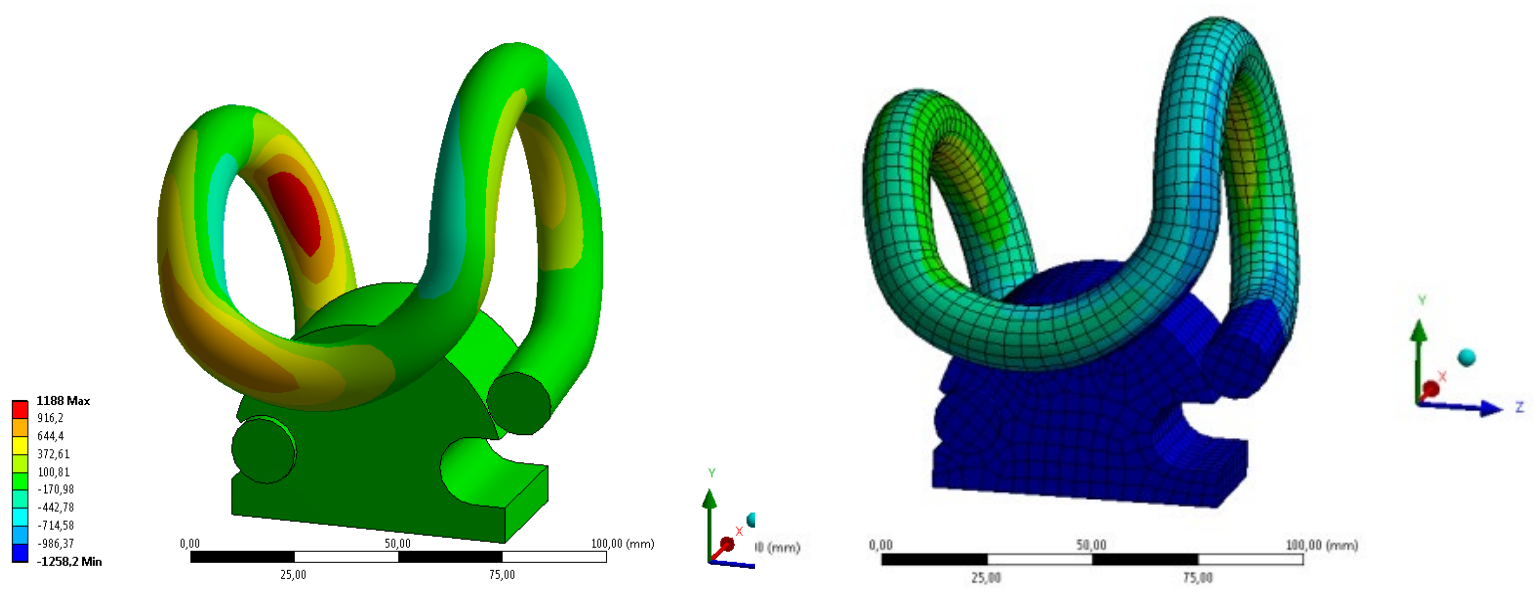

Fig. 10. Contours of the: a) stress difference $\left(\sigma_{\mathrm{y}}-\sigma_{\mathrm{z}}\right)$ in final clip fastening stage, $\left.b\right)$ reduced stress $\sigma_{\text {eq }}$ (von Misesa)

The simulations performed confirmed the acceptable level of effort of the SB4 clip material during its fastening: $\sigma_{e q}<R_{e}$.

\section{Summary}

The above-mentioned experimental tests and numerical calculations were performed for the critical load case of the SB4 clip. The obtained results confirmed the acceptable level of effort of the clip material during its fastening, with the occurrence of complex states of temporarily high stress states being the characteristic feature of this process.

\section{References}

[1] Information on http://www.pandrol.com/application/high-speed/

[2] Information on http:// www.railway-fasteners.com

[3] Information on http:// www.pandrol.com/product/pandrol-fastclip-fd/

[4] Information on http:// www.vossloh.com/en/

[5] Information on https://publications.europa.eu, Commission Regulation (EU) No 1299/2014 of 18 November 2014 on the technical specifications for interoperability relating to the "infrastructure" subsystem of the rail system in the European Union

[6] Instructional material provided by Vossloh (in polish). (2016)

[7] J.W. Dally, Experimental Stress Analysis, third ed., McGraw-Hill, Inc., New York, 1991.

[8] A. S. Khan, X.Wang, Strain Measurements and Stress Analysis, first ed., Prentice Hall, 2001. 